\title{
Severe outcomes and strategies for the prediction and treatment in pregnancies with pernicious placenta previa and prior cesarean delivery - a national retrospective study
}

\author{
Guiqin Bai ${ }^{1}$, Weilin Chen ${ }^{2}$, Xianghua Huang ${ }^{3}$, Shaojie Zhao ${ }^{4}$, Shuping Zhao ${ }^{5}$, Xiujuan \\ $\mathrm{Chen}^{6}$, Suwen Chen ${ }^{7}$, Hua Yang ${ }^{8}$, Xia Lu ${ }^{9}$, Guanyuan Liu ${ }^{10}$, Qionghua Chen ${ }^{11}$, Linai \\ Zhang ${ }^{12}, \mathrm{Li}^{\mathrm{Jin}}{ }^{2}$, and Yongzheng $\mathrm{Wu}^{13}$ \\ ${ }^{1}$ Xi'an Jiaotong University Medical College First Affiliated Hospital \\ ${ }^{2}$ Peking Union Medical College Hospital \\ ${ }^{3}$ Hebei Medical University No2 Hospital \\ ${ }^{4}$ Nanjing Medical University \\ ${ }^{5}$ Qingdao Women and Children's Hospital \\ ${ }^{6}$ Fujian Province Maternal and Child Health Hospital \\ ${ }^{7}$ Capital Medical University Beijing Obstetrics and Gynecology Hospital \\ ${ }^{8}$ Tianjin Central Hospital of Obstetrics and Gynecology \\ ${ }^{9}$ Xin Jiang Medical University \\ ${ }^{10}$ Beijing Chaoyang Hospital \\ ${ }^{11}$ Xiamen University and Fujian Medical University Affiliated First Hospital \\ ${ }^{12}$ Shanxi Maternal and Child Health Hospital \\ ${ }^{13}$ Institut Pasteur
}

August 28, 2020

\begin{abstract}
Objectives To evaluate the severe outcomes in pregnancies with pernicious placenta previa and prior cesarean delivery, and to analyze the predictive examinations and prevention strategies regarding severe complications in these women. Design Multicentre retrospective cohort study. Setting Thirteen hospitals in China. Population Selected 747 women with pernicious placenta previa and prior cesarean history. Methods The basic data relating to pregnancy, the examinations during gestation and the outcomes were collected and analyzed. Main outcome measures Outcomes including massive bleeding, placenta implantation and hysterectomy were monitored. Results Our results showed that placental implantation occurred in $47.5 \%$ of patients and the hysterectomy rate was $10.4 \%$. The incidence of massive bleeding and blood transfusion was $55.8 \%$ and $64 \%$, respectively. The women with placenta implantation predicted by both ultrasound and MRI had higher blood loss than those diagnosed only by either one of the examinations. Surprisingly, vascular occlusion had no effect on the occurrence of severe hemorrhage and hysterectomy. The latter was even higher in the vascular blocking group compared to that in the non-blocking group ( $34.2 \%$ and $8.6 \%$, respectively). Conclusions: The pregnancies with pernicious placenta previa and prior cesarean delivery, had a dramatically higher risk of placenta implantation, hysterectomy and massive hemorrhage. MRI combined with ultrasound examination presented high accuracy in predicting severe outcomes in these patients. Vascular occlusion does not appear to be an effective approach to prevent severe outcomes. Surgical hemostasis should be the key goal in blocking massive bleeding, preserving the uterus and improving the prognosis of the patients.
\end{abstract}

Severe outcomes and strategies for the prediction and treatment in pregnancies with pernicious 


\section{placenta previa and prior cesarean delivery - a national retrospective study}

Guiqin Bai ${ }^{1}$, Weilin Chen ${ }^{2}$, Xianghua Huang ${ }^{3}$, Shaojie Zhao ${ }^{4}$, Shuping Zhao ${ }^{5}$, Xiujuan Chen ${ }^{6}$, Suwen Chen ${ }^{7}$, Hua Yang ${ }^{8}$, Xia Lu ${ }^{9}$, Guanyuan Liu ${ }^{10}$, Qionghua Chen ${ }^{11}$, Lin'ai Zhang ${ }^{12}$, Yongzheng Wu ${ }^{13^{*}}$, Li Jin ${ }^{2 *}$

${ }^{1}$ Department of Gynecology and Obstetrics, the First Affiliated Hospital, Xi'an Jiaotong University, Xi'an City, China. ${ }^{2}$ Department of Gynecology and Obstetrics, Peking Union Medical College Hospital, Beijing City, China. ${ }^{3}$ Department of Gynecology and Obstetrics, the Second Hospital of Hebei University, Shijiazhuang City, China. ${ }^{4}$ Department of Gynecology, Wuxi Maternal and Child Health Hospital, the Affiliated Hospital of Nanjing Medical University, Wuxi City, China. ${ }^{5}$ Department of Gynecology, Qingdao Women \&Children Hospital, Qingdao City, China. ${ }^{6}$ Department of Family Planning, Fujian Provincial Maternity and Children's Hospital, Fuzhou City, China. ${ }^{7}$ Department of Family Planning, Beijing Obstetrics and Gynecology Hospital, Capital Medical University, Beijing City, China. ${ }^{8}$ Department of Family Planning, Tianjin Central hospital of Gynecology and obstetrics, Tianjin City, China. ${ }^{9}$ Department of Gynecology and Obstetrics, the Sixth Affiliated Hospital of Xinjiang Medical University, Urumchi City, China. ${ }^{10}$ Department of Gynecology and Obstetrics, Beijing Chaoyang Hospital,Capital Medical University, Beijing City, China. ${ }^{11}$ Department of Gynecology and Obstetrics, the First Affiliated Hospital of Xiamen University, Xiamen City, China. ${ }^{12}$ Department of Family Planning, Shanxi Children's Hospital (Shanxi Maternal and Child Health Hospital), Taiyuan City, China. ${ }^{13}$ Unit of Cellular Biology \& Microbial Infection, CNRS UMR3691, Institut Pasteur, Paris, France.

Short title : Outcomes in pregnancies with placenta previa

*Correspondent authors

Yongzheng $\mathrm{Wu}$

Unit de Biologie Cellulaire de Infection Microbienne, CNRS UMR3691, Institut Pasteur, Paris, France

Tel : 33-140613062

Fax: 33-140613238

Email : wuyzh@pasteur.fr

Li Jin, $\mathrm{PhD}$

Peking Union Medical College Hospital, Peking Union Medical College, Chinese Academy of Medical Sciences, Beijing, China

Tel: 86-13611282080.

Email:jinlipumch@hotmail.com.

\section{ABSTRACT}

\section{Objectives}

To evaluate the severe outcomes in pregnancies with pernicious placenta previa and prior cesarean delivery, and to analyze the predictive examinations and prevention strategies regarding severe complications in these women.

\section{Design}

Multicentre retrospective cohort study.

\section{Setting}

Thirteen hospitals in China.

\section{Population}

Selected 747 women with pernicious placenta previa and prior cesarean history. 


\section{Methods}

The basic data relating to pregnancy, the examinations during gestation and the outcomes were collected and analyzed.

\section{Main outcome measures}

Outcomes including massive bleeding, placenta implantation and hysterectomy were monitored.

\section{Results}

Our results showed that placental implantation occurred in $47.5 \%$ of patients and the hysterectomy rate was $10.4 \%$. The incidence of massive bleeding and blood transfusion was $55.8 \%$ and $64 \%$, respectively. The women with placenta implantation predicted by both ultrasound and MRI had higher blood loss than those diagnosed only by either one of the examinations. Surprisingly, vascular occlusion had no effect on the occurrence of severe hemorrhage and hysterectomy. The latter was even higher in the vascular blocking group compared to that in the non-blocking group ( $34.2 \%$ and $8.6 \%$, respectively).

Conclusions: The pregnancies with pernicious placenta previa and prior cesarean delivery, had a dramatically higher risk of placenta implantation, hysterectomy and massive hemorrhage. MRI combined with ultrasound examination presented high accuracy in predicting severe outcomes in these patients. Vascular occlusion does not appear to be an effective approach to prevent severe outcomes. Surgical hemostasis should be the key goal in blocking massive bleeding, preserving the uterus and improving the prognosis of the patients.

Funding statement: This study was supported by the Innovative Team Program of Shaanxi Province (project $\mathrm{N}^{\circ}:$ 2019TD-031).

Key words : cesarean section; pernicious placenta previa; massive bleeding; placental implantation; hysterectomy, vascular occlusion, severe outcomes

\section{TWEETABLE ABSTRACT}

Severe bleeding and placental implantation are main outcomes in pregnancies with pernicious placenta previa and prior cesarean, and vascular occlusion does not block massive bleeding.

\section{INTRODUCTION}

Placenta previa often occurs in pregnant women who have had increased age, multiple gestations, high parity, and smoke or use illegal drugs (1). Cesarean delivery induced scar formation in uterine and endometrial injuries also facilitates the development of placenta previa in the subsequent pregnancies of women $(2,3)$. It is estimated that placenta previa occurs in $0.28-0.55 \%$ of pregnant women in the USA (4). The overall incidence of placenta previa in China has recently been reported to represent $1.24 \%$ of deliveries (5). Placenta previa can cause severe maternal and neonatal complications including the limitation of fetal growth, preterm birth, hemorrhage whatever the degree of overlying placenta and gestation stage, hysterectomy and even the mortality of the fetus and mother $(6,7)$.

To avoid these consequences, cesarean section has been widely applied in pregnancies with placenta previa. However, despite its important role in managing dystocia, pregnancy complications, and reducing maternal and child mortality and morbidity, cesarean delivery rates have increased around the world $(8,9)$. For example, in China, it increased from about $5 \%$ in the 1960 s to $20 \%$ in the late 1980 s and early 1990 s (10). In particular, cesarean sections made of $40-60 \%$ of deliveries in most hospitals in China during the last 20 years $(11,12)$. On the other hand, cesarean history increases the risk of placenta previa by $60 \%$ in the subsequent pregnancy of women (13). A pregnant woman who has both a complete placenta previa and cesarean history is more likely to have high risk of placenta implantation, severe bleeding during gestation and delivery, and massive postpartum hemorrhage $(14,15)$. More seriously, in the case where the placenta grows on the scar of a previous cesarean section, referred to as pernicious placenta previa, increases the risk of placenta implantation by up to $50 \%$ (16). Both pernicious placenta previa and placenta implantation are 
very critical and urgent situations in obstetrics, in which fatal bleeding occurs very often during delivery, and the maternal mortality rate is as high as $7 \%$ (17). However, no collective data have been reported up to now to demonstrate the severe outcomes and associated risk factors in patients with pernicious placenta previa. To prevent severe bleeding during delivery and severe postpartum hemorrhage in these patients, vascular occlusion has been widely used in clinical practice, with methods such as bilateral uterine artery embolization (UAE), internal iliac artery embolization (IIAE) and intra-aortic balloon occlusion $(18,19)$, although their anti-bleeding effect and influence on the prognosis of pregnancies remain unclear.

We performed a retrospective study in which data on 747 patients who had pernicious placenta previa in their pregnancy following their first cesarean delivery, were collected from 13 first-class hospitals located in different regions of China. The severe consequences and associated risk factors of these patients were analyzed. In particular, we evaluated the approaches taken to predict severe outcomes and the influence of vascular occlusion in preventing severe postpartum hemorrhage and hysterectomy.

\section{OBJECTS AND METHODS}

\section{Patients}

This nationwide retrospective study was performed with 747 patients recruited from 13 first-class hospitals located in the different regions of mainland China. The criteria used for patient selection was: previous history of cesarean section; cesarean delivery for this time; edged, partial or complete placenta previa; placenta previa growing on the uterine scar of previous cesarean surgery. The women who had lower placenta or edged placenta previa in the posterior or lateral wall, were excluded. A hundred and six of these patients, who received uterine artery embolization or abdominal aortic balloon occlusion, were assigned to the blocking group. The other 641 women were considered as the non-blocking group.

\section{Diagnosis of placenta previa and placenta implantation}

In the present study, placenta previa was diagnosed by ultrasound and/or magnetic resonance (MRI) examination during the regular follow-up no matter whether the placenta reached, or partially or completely covered the opening of the endo-cervix. The final diagnosis of placenta previa was confirmed during the cesarean surgery.

The placenta implantation was predicted by ultrasound and/or MRI, or determined directly if it was difficult to remove during the operation, together with subsequent pathological examination.

\section{Data collection}

The basic information on the pregnant women was collected, including age, the times of pregnant, delivery times ([?] 28 weeks of delivery), the numbers of former caesarean, the inter-birth interval between the previous and the current caesarean section, the duration of gestation, the thickness of the uterine scar, the type of placenta previa under ultrasound (marginal, partial or complete at the front wall of the uterus) and the condition of placenta attachment (adhesion, implantation or penetration).

The data for perinatal outcomes of mother and newborn were also collected, including the termination time of the pregnancy, whether arterial blocking was applied and the related method, postpartum hemorrhage, the volume of bleeding during the delivery, blood transfusion, hysterectomy, uterine rupture, the intraoperative situation of the placenta, infection, admission to an intensive care unit, intraoperative hemostatic operations and the medicine used, the fetal biparietal diameter before delivery, and Apgar score at 1min and 5min after birth of newborns.

\section{Statistical analysis}

The software SPSS 24.0 was used for the data analysis. The median (range/lower quartile and upper quartile, M-Range/QL \& QU) was used to describe its concentration and tendency of dispersion, since not all the quantitative data were shown as the normal distribution. 
The Kruskal-Wallis $\mathrm{H}$ test was used for the comparison between multiple independent groups. The enumeration data were represented as rate or composition ratio and the chi-square test was applied for comparisons between groups. The binary non-conditional logistic regression with single factor and multi-factor analyses were conducted to check the influencing factors of related variables. The predominant factors from the single factor analysis were considered for the further multi-factor regression analysis. The forward stepwise regression method was also adopted in the present study and $\mathrm{p}$ values lower than 0.05 and 0.10 were used as criteria. The two-tailed test was applied in all the analyses and $\mathrm{p}<0.05$ was considered significant. The $\mathrm{p}$ value for the comparison of two groups was modified to 0.017 .

\section{RESULTS}

The severe outcomes of pregnancies with pernicious placenta previa after the first cesarean delivery

In this study, the clinical consequences such as hysterectomy, severe bleeding during delivery and massive postpartum hemorrhage $([?] 1000 \mathrm{ml})$, and placental implantation, were defined as severe outcomes. Our result showed that the gestation of pregnancies was $30-39^{+5}$ weeks. As shown in Fig. 1, the hysterectomy was conducted in 78 cases with hysterectomy rate of $10.4 \%$. The uterine rupture rate was $0.8 \%$. The placenta implantation was confirmed from 355 women during operation with incidence rate of $47.5 \%$. Meanwhile, $55.8 \%$ of pregnancies had massive hemorrhage. The blood transfusion was given to $64 \%$ of pregnancies. No maternal or fetal mortality was reported.

Analysis of the risk factors associated with the severe complications in these patients

We next analyzed the risk factors associated with these severe outcomes using binary non-conditional logistic regression analysis with single factor. Eight factors potentially associated with the consequence of hysterectomy $(\mathrm{p}<0.05)$, including placenta implantation detected by ultrasound and/or MRI, placenta previa type, application of vascular occlusion and type of vascular blocking, postpartum hemorrhage ([?]1000ml), bleeding volume during the delivery, and confirmed placenta implantation by the surgery. These factors were further analyzed by the non-conditional binary logistic regression with multi-factors. As shown in Table S1, 5.319 times higher potential of hysterectomy was found in the patients with uterine artery embolization than those with abdominal aortic balloon occlusion. This probability was almost doubled (1.002 times) with every $1 \mathrm{ml}$ increase of bleeding during the delivery, relevant to that before bleeding. It suggested that the mode of vascular embolization and the volume of hemorrhage during delivery were the main risk factors associated with hysterectomy.

Similarly, 7 factors were identified by single factor analysis, which were associated with severe bleeding during the delivery, as shown in Table 1. Evaluation of these risks by multifactor regression analysis showed that the pregnancies with [?]2 cesarean deliveries had higher possibility (3.562 times) of severe bleeding than those with only once. Compared to women without placenta implantation, patients diagnosed or suspected placenta implantation by ultrasound had higher potential (1.631 times, 95\% CI:1.000-2.658 or 1.794 times, 95\% CI:1.110-2.899) of severe bleeding. Particularly, this probability increased 6.839 and 1.964 times in the pregnancy with confirmed or suspected placenta implantation by surgery than those without implantation (95\% CI:4.508-10.377 or 1.259-3.064), respectively. Complete placenta previa also resulted in 1.814 times severe bleeding in patients than marginal one (95\% CI:1.219-2.698). These results suggested that cesarean numbers, ultrasound-detected or surgery-confirmed placenta implantation, and type of placenta previa were associated tightly with massive bleeding during the delivery.

Our analysis also demonstrated that under ultrasound every $0.1 \mathrm{~cm}$ increase of the thickness of uterine scar, promoted the likelihood of placenta implantation to 1.559 times (95\% CI:1.205-2.017). Suspected placenta implantation under ultrasound than normal placenta had 7.79 times high likelihood of implantation confirmed later by surgery (95\% CI:1.526-39.765). The subjects without MRI examination also had 3.565 times higher probability of placenta implantation than those with non-implantation diagnosed by MRI (95\% CI:1.622-7.837). A higher potential of placenta implantation (3.704 times) was also observed in the women with complete placenta previa than marginal one (95\% CI:1.699-8.076). These evidences indicated that ultrasound-detected thickness of uterus scar, ultrasound and/or MRI-detected or suspected implantation of 
placenta, and complete placenta previa, were closely associated with placenta implantation.

Role of ultrasound and MRI examination in predicting placental abnormality and severe outcomes of pregnancies

All 747 pregnancies took ultrasound examination by which abnormal placental adhesion were detected from 271 of them, while placenta implantation was confirmed from 514 pregnancies by surgery, including 244 cases who had ultrasound-diagnosed abnormal placenta (Table 2). It suggested that the predictive sensitivity and specificity by ultrasound was $47.5 \%$ and $88.4 \%$, respectively. In addition, misdiagnosis was $11.6 \%(27 / 233$ cases) and missed diagnosis was $52.5 \%$ (270/514 cases).

Placenta implantation was predicted in 143 subjects taken MRI, and 141 of whom were confirmed later by surgery, showing $79.2 \%$ of sensitivity and $97.8 \%$ of specificity for MRI-predicted placental implantation (Table 2). The rate of misdiagnosis and missed diagnosis was 2.2\% (2/90 cases) and 20.8\% (37/178 cases), respectively (Table 2). Intriguingly, 56 patients had both predicted- (during gestation by both ultrasound and MRI) and confirmed- (intra-operation) placenta implantation, indicating $100 \%$ of specificity. But, 122 surgery-confirmed placenta implantations were not detected by both examinations with $68.5 \%$ of missed diagnosis. In addition, 154 placenta implantations detected only by either of examinations were confirmed later by surgery with a higher sensitivity (86.5\%). All these results revealed the essential role of ultrasound and MRI in detecting placental abnormality during the gestation.

We also analyzed the relationship between ultrasound- and/or MRI-predicted placenta implantation and other adverse outcomes in patients. As shown in Table 3, bleeding volume during delivery, volume of blood transfusion and hysterectomy were tightly associated with placenta implantation no matter whether implantation was predicted by ultrasound and/or MRI. The comparison between any two conditions $\left(\alpha^{\prime}=0.017\right)$ demonstrated that the amount of hemorrhage and blood transfusion in the implantation-predicted groups were bigger than that in the non-implanted group. The patients with placenta implantation detected by both ultrasound and MRI, also had significant increase of hemorrhage volume, blood transfusion and hysterectomy than those by either of examinations.

Role of vascular occlusion in the consequence of women with pernicious placenta previa and prior cesarean section

Vascular occlusion has been applied to reduce bleeding during the delivery (20-23), which was verified in this study. Vascular occlusion was applied to 106 pregnancies by either uterine artery embolization or abdominal aorta balloon occlusion (blocking group). The other 641 cases were assigned as unblocked group. The basic information of patients in two groups was described in Table S2 and no significant difference was observed between two groups. However, more suspected placenta implantation was observed by ultrasound in the blocking group (26.4\%) than that in the non-blocking group (15.9\%). The bleeding volume during the delivery (600 2500 vs . $5002000 \mathrm{ml}, \mathrm{p}=0.093)$ and the incidence of postpartum hemorrhage (63/106 vs 354/641 cases, $\mathrm{p}=0.419)$ were similar between blocking and non-blocking group. Surprisingly, the hysterectomy rate in blocking group $(25.4 \%, 27 / 106$ cases $)$ was much higher than that in non-blocking patients $(7.9 \%, 51 / 641$ cases) $(\mathrm{p}<0.001)$. The effect of vascular occlusion on the fetal outcomes was also checked in this study. No differences were detected between blocking and non-blocking group, including the gestation terms of pregnancies and the Apgar score after birth (1 \& 5 minutes) (Table S3).

\section{COMMENT}

\section{Main findings}

The women who had pernicious placenta previa in the subsequent pregnancy after first cesarean delivery, had a higher frequency of severe outcomes including placental implantation (47.5\%), massive bleeding $(55.8 \%)$ and hysterectomy (10.4\%). The examination of ultrasound combined with MRI improved the prediction of placenta implantation in these patients. Finally, we showed that vascular occlusion had no effect on the occurrence of severe hemorrhage and hysterectomy. 


\section{Strengths and limitations}

Among the similar retrospective studies, to our knowledge, this study is the largest cohort with 747 women who gives subsequent cesarean birth with placenta previa after the first cesarean delivery, since this kind of medical situation is rare in clinic, but, with life-threatening consequences. These patients were from 13 hospitals located in the different regions of China. Although these hospitals belong to the first-class hospitals in China and represent the top level of local medical care, the regional differences on medical care are existed among these hospitals, which may cause the variation of the outcome occurrence and treatment, and can be considered as flaw for the present study. However, the obtained conclusions are still national representative. Another key finding in our study is that the examination of ultrasound combined with MRI largely improves the prediction of potential outcome in the patients, which can used to follow up patients in order to prevent and treat the potential severe complications. Finally, we also demonstrate that vascular occlusion does not affect massive bleeding in patients, which is disagree with the current opinion. But, we have to point out that vascular occlusion in the current study is performed only in 106 patients, among whom the different strategies for vascular blocking is applied, which is another limitation for this study and more cases and prospective study are needed to confirm our conclusion.

\section{Interpretation}

Previous cesarean history and current pregnancy with placenta previa can lead to placenta growth on the scar tissue of cesarean surgery, which is often accompanied with placenta implantation, resulting in unpredictable and uncontrollable severe outcomes (24). Unfortunately, it is quite difficult to precisely diagnose placental growth on the scar tissue of the uterus and placenta implantation. Meanwhile, this kind of pregnancies, in particular, with pernicious placenta previa in the subsequent pregnancy as in our study, are clinically rare and always had severe outcomes including massive bleeding, hysterectomy and even maternal and fetal mortality. However, up to now no national or international guideline has been published for the diagnosis and treatment of this critical situation in pregnant women, although a few reports have been published with very limited case numbers $(25,26)$.

Among the 747 pregnancies in this study, $55.8 \%$ and $47.5 \%$ of them had massive bleeding and placenta implantation, respectively. Uterine rupture occurred in $0.8 \%$ of those pregnancies and $10.4 \%$ required hysterectomy. On the contrary, hysterectomy $(22.4 \%)$ was reported as the most common severe complication in the pregnancies with placenta previa and prior cesarean delivery (27). The present study also found that MRI examination has higher sensitivity and specificity in determining placenta implantation than ultrasound. In line with our observation, MRI has been proposed for placenta implantation diagnosis due to its characteristics of wide field of vision, high resolution and contrast for the soft tissue $(28,29)$. Importantly, placenta implantation predicted by both ultrasound and MRI, was confirmed later by the surgery. These pregnancies (56 cases) with 'double-prediction' of placenta implantation, also had a higher amount of bleeding during surgery and hysterectomy potential than women with MRI- or ultrasound-predicted placenta implantation alone. Therefore, MRI combined with ultrasound examination enabled not only to better determination of placenta implantation, but also the prediction of the outcomes of pregnancy.

The application of vascular intervention in obstetrics significantly decreased hysterectomy rate, blood loss and transfusion, and admission to intensive care unit (30-36), particularly, in the pregnancies with placenta previa and prior cesarean delivery $(37,38)$. Surprisingly, our results showed that the estimated blood loss during delivery had no difference between blocking and non-blocking group, and that the blocking group even had a higher hysterectomy rate than the non-blocking group. To support our observation, abdominal aortic balloon was reported to block artery circulation and reduce bleeding temporarily, and re-bleeding occurred once balloon was removed (39). Another study also showed that abdominal aortic balloon could not effectively reduce the blood loss in the patients with placenta implantation compared to the non-blocking group (40). The potential reason for this opposite conclusion between ours and others is that all other studies have a small number of patients, while 747 pregnancies from 13 first-class hospitals located in the different regions of China were included in this study. Combined these evidences, the published data and related complications $(29,41,42)$, we conclude that the effect of vascular blocking approach in blocking massive 
bleeding during the delivery indeed needs to be deliberated.

\section{Conclusions}

Our study elucidated that massive bleeding and placenta implantation were the main severe adverse complications in pregnancies with pernicious placenta previa and prior cesarean history. The examination of MRI together with ultrasound during the routine follow-up obviously improved the diagnosis of placenta implantation in these patients. However, vascular occlusion is probably not an effective approach for preventing severe outcomes in these pregnancies. We suggest that surgical hemostasis is the key issue in blocking severe bleeding, preserving the uterus and improving the prognosis of patients. These characteristics should be considered by physicians for this kind of patient in the future.

Disclosure of Interest : The author(s) report(s) no conflict of financial, personal, political, intellectual or religious interests.

\section{Author's Contribution:}

GQB contributed to study conception and design, data analysis and participation in writing first draft.

WLC contributed to the data collection.

XHH contributed to the data collection.

SJZ contributed to the data collection.

XJC contributed to the data collection.

SWC contributed to the data collection.

HY contributed to the data collection.

XL contributed to the data collection.

GYL contributed to the data collection.

QHC contributed to the data collection.

LZ contributed to the data collection.

YZW contributed to the manuscript writing.

LJ contributed to study conception and design, participated in writing first draft.

\section{Details of Ethics Approval:}

The ethics approval (approval N: XJTU1AF2018LSL-3D005) was obtained on January 8, 2019 from the Ethics Committee at the Affiliated First Hospital of Xi'an Jiaotong University.

\section{Funding:}

This study was supported by the Innovative Team Program of Shaanxi Province (project N: 2019TD-031).

\section{REFERENCES}

1. Faiz AS, Ananth CV. Etiology and risk factors for placenta previa: an overview and meta-analysis of observational studies. J Matern-Fetal Neonatal Med Off J Eur Assoc Perinat Med Fed Asia Ocean Perinat Soc Int Soc Perinat Obstet. mars 2003;13(3):175-90.

2. Mastrolia SA, Baumfeld Y, Loverro G, Yohai D, Hershkovitz R, Weintraub AY. Placenta previa associated with severe bleeding leading to hospitalization and delivery: a retrospective population-based cohort study. J Matern-Fetal Neonatal Med Off J Eur Assoc Perinat Med Fed Asia Ocean Perinat Soc Int Soc Perinat Obstet. nov 2016;29(21):3467-71. 
3. Bender S. Placenta previa and previous lower segment cesarean section. Surg Gynecol Obstet. mai 1954;98(5):625-8.

4. Wortman AC, Schaefer SL, McIntire DD, Sheffield JS, Twickler DM. Complete Placenta Previa: Ultrasound Biometry and Surgical Outcomes. AJP Rep. avr 2018;8(2):e74-8.

5. Fan D, Wu S, Wang W, Xin L, Tian G, Liu L, et al. Prevalence of placenta previa among deliveries in Mainland China: A PRISMA-compliant systematic review and meta-analysis. Medicine (Baltimore). oct 2016;95(40):e5107.

6. Crane JM, Van den Hof MC, Dodds L, Armson BA, Liston R. Maternal complications with placenta previa. Am J Perinatol. 2000;17(2):101-5.

7. Ananth CV, Demissie K, Smulian JC, Vintzileos AM. Relationship among placenta previa, fetal growth restriction, and preterm delivery: a population-based study. Obstet Gynecol. aout 2001;98(2):299-306.

8. Boerma T, Ronsmans C, Melesse DY, Barros AJD, Barros FC, Juan L, et al. Global epidemiology of use of and disparities in caesarean sections. Lancet Lond Engl. 13 2018;392(10155):1341-8.

9. Roberts CL, Nippita TA. International caesarean section rates: the rising tide. Lancet Glob Health. mai 2015;3(5):e241-242.

10. Qin C, Zhou M, Callaghan WM, Posner SF, Zhang J, Berg CJ, et al. Clinical Indications and Determinants of the Rise of Cesarean Section in Three Hospitals in Rural China. Matern Child Health J. 1 oct 2012;16(7):1484-90.

11. Wang X, Hellerstein S, Hou L, Zou L, Ruan Y, Zhang W. Caesarean deliveries in China. BMC Pregnancy Childbirth [Internet]. 6 fevr 2017 [cite 27 fevr 2020];17. Disponible sur: https://www.ncbi.nlm.nih.gov/pmc/articles/PMC5294866/

12. weiyuan Z, Hou L. Enlightenment of big data survey on cesarean section status in mainland China. Chin J Pract Gynecol Obstet. 2018;34:38-40.

13. Gurol-Urganci I, Cromwell DA, Edozien LC, Smith GCS, Onwere C, Mahmood TA, et al. Risk of placenta previa in second birth after first birth cesarean section: a population-based study and meta-analysis. BMC Pregnancy Childbirth. 21 nov 2011;11:95.

14. WANG Y, ZHONG L, ZHONG S, YU Z. Ultrasonographic features of placenta previa with placenta implantation. Journal of Medical Imaging. 2017;27:1533-6.

15. B.Tutschek* F.Chantraine* T.Braun* W.Henrich. Sonographische Diagnos-tik bei abnorm invasiver Plazenta. 2014;47:408-17.

16. Chattopadhyay SK, Kharif H, Sherbeeni MM. Placenta praevia and accreta after previous caesarean section. Eur J Obstet Gynecol Reprod Biol. 30 dec 1993;52(3):151-6.

17. Clausen C, Lonn L, Langhoff-Roos J. Management of placenta percreta: a review of published cases. Acta Obstet Gynecol Scand. fevr 2014;93(2):138-43.

18. He Q, Li Y-L, Zhu M-J, Peng X-C, Liu X-Y, Hou H-L, et al. Prophylactic abdominal aortic balloon occlusion in patients with pernicious placenta previa during cesarean section: a systematic review and metaanalysis from randomized controlled trials. Arch Gynecol Obstet. nov 2019;300(5):1131-45.

19. Zhou X, Sun X, Wang M, Huang L, Xiong W. The effectiveness of prophylactic internal iliac artery balloon occlusion in the treatment of patients with pernicious placenta previa coexisting with placenta accreta. J Matern-Fetal Neonatal Med Off J Eur Assoc Perinat Med Fed Asia Ocean Perinat Soc Int Soc Perinat Obstet. 9 avr 2019;1-6.

20. Manzano-Nunez R, Escobar-Vidarte MF, Naranjo MP, Rodriguez F, Ferrada P, Casallas JD, et al. Expanding the field of acute care surgery: a systematic review of the use of resuscitative endovascular 
balloon occlusion of the aorta (REBOA) in cases of morbidly adherent placenta. Eur J Trauma Emerg Surg Off Publ Eur Trauma Soc. aout 2018;44(4):519-26.

21. Kwon Y-S, Cho YM, Im KS, Yoo SB, Hyung SW. Transient Occlusion of Uterine Arteries in Procedures with High Risk of Uterine Bleeding. JSLS. mars 2019;23(1).

22. Sun L. Application of interventional treatment on dangerous placenta praevia complicated with placenta accrete. Chin J Fam Plan Gynecotokology. 2018;3:50-2.

23. LIU Z, ZHAO X, LIU C, Wang Y, Zhang W, Wang X, et al. The Application of Temporary Ballon Occlusion of the Abdominal Aorta in Patients with Pernicious Placenta Previa and Placenta Accreta. J Pract Obstet Gynecol. 32:204-7.

24. Wei LC, Gong GY, Chen JH, Hou PY, Li QY, Zheng ZY, et al. [Application of lower abdominal aorta balloon occlusion technique by ultrasound guiding during caesarean section in patients with pernicious placenta previa]. Zhonghua Yi Xue Za Zhi. 27 mars 2018;98(12):930-4.

25. Li M, Du F, Teng Y, Huang Y, Tao M, Gu J, et al. The Pregnancy Outcome and the High Risk Factor of Postpartum Hemorrhage in PPP Patients. Journal of Practical Obstetrics and Gynecology. 2017;33:755-9.

26. Li S, Du D, Wang L. The correlation between placental attachment and dangerous postpartum hemorrhage of placenta previa and its risk. Chinese Journal of Difficult and Complicated Cases. 18:388-91.

27. Zhou M, Chen M, Zhang L, He G-L, He L, Wei Q, et al. [Severe Adverse Pregnancy Outcomes in Placenta Previa and Prior Cesarean Delivery]. Sichuan Da Xue Xue Bao Yi Xue Ban. sept 2017;48(5):783-7.

28. Aitken K, Allen L, Pantazi S, Kingdom J, Keating S, Pollard L, et al. MRI Significantly Improves Disease Staging to Direct Surgical Planning for Abnormal Invasive Placentation: A Single Centre Experience. J Obstet Gynaecol Can JOGC J Obstet Gynecol Can JOGC. 2016;38(3):246-251.e1.

29. Lv C. Clinical Analysis of Implantable Severe Placenta Previa Using Magnetic Resonance. China \& Foreign Medical Treatment. :2016,35(05);169-170.

30. Panici PB, Anceschi M, Borgia ML, Bresadola L, Masselli G, Parasassi T, et al. Intraoperative aorta balloon occlusion: fertility preservation in patients with placenta previa accreta/increta. J Matern-Fetal Neonatal Med Off J Eur Assoc Perinat Med Fed Asia Ocean Perinat Soc Int Soc Perinat Obstet. dec 2012;25(12):2512-6.

31. Harma M, Harma M, Kunt AS, Andac MH, Demir N. Balloon occlusion of the descending aorta in the treatment of severe post-partum haemorrhage. Aust N Z J Obstet Gynaecol. avr 2004;44(2):170-1.

32. Bell-Thomas SM, Penketh RJ, Lord RH, Davies NJ, Collis R. Emergency use of a transfemoral aortic occlusion catheter to control massive haemorrhage at caesarean hysterectomy. BJOG Int J Obstet Gynaecol. dec 2003;110(12):1120-2.

33. Paull JD, Smith J, Williams L, Davison G, Devine T, Holt M. Balloon occlusion of the abdominal aorta during caesarean hysterectomy for placenta percreta. Anaesth Intensive Care. dec 1995;23(6):731-4.

34. Andoh S, Mitani S, Nonaka A, Suzuki S, Tamaki F, Ohmori K, et al. [Use of temporary aortic balloon occlusion of the abdominal aorta was useful during cesarean hysterectomy for placenta accreta]. Masui. fevr 2011;60(2):217-9.

35. Liu HY, Lin XG, Wu JL, Qiao FY, Deng DR, Zeng WJ. [Multiposition spiral suture of the lower uterine segment: a new technique to control the intraoperative bleeding of pernicious placenta previa]. Zhonghua Fu Chan Ke Za Zhi. 25 oct 2016;51(10):754-8.

36. Du X, Xie X, Wang Y. Uterine artery suture: a preventive approach for pernicious placenta previa. Cell Biochem Biophys. mars 2014;68(2):407-10. 
37. Wei LC, Gong GY, Chen JH, Hou PY, Li QY, Zheng ZY, et al. [Application of lower abdominal aorta balloon occlusion technique by ultrasound guiding during caesarean section in patients with pernicious placenta previa]. Zhonghua Yi Xue Za Zhi. 27 mars 2018;98(12):930-4.

38. Dai M-J, Jin G-X, Lin J-H, Zhang Y, Chen Y-Y, Zhang X-B. Pre-cesarean prophylactic balloon placement in the internal iliac artery to prevent postpartum hemorrhage among women with pernicious placenta previa. Int J Gynaecol Obstet Off Organ Int Fed Gynaecol Obstet. sept 2018;142(3):315-20.

39. Xu J-Q. Effectiveness of embolization of the internal iliac or uterine arteries in the treatment of massive obstetrical and gynecological hemorrhages. Eur Rev Med Pharmacol Sci. 2015;19(3):372-4.

40. Qi X-R, Liu X-H, You Y, Wang X-D, Zhou R, Xing A-Y, et al. [The Application of Internal Iliac Artery Balloon Occlusion in Pernicious Placenta Previa]. Sichuan Da Xue Xue Bao Yi Xue Ban. juill 2016;47(4):618-23.

41. Qi X-R, Liu X-H, You Y, Wang X-D, Zhou R, Xing A-Y, et al. [The Application of Internal Iliac Artery Balloon Occlusion in Pernicious Placenta Previa]. Sichuan Da Xue Xue Bao Yi Xue Ban. juill 2016;47(4):618-23.

42. Cali G, Forlani F, Giambanco L, Amico ML, Vallone M, Puccio G, et al. Prophylactic use of intravascular balloon catheters in women with placenta accreta, increta and percreta. Eur J Obstet Gynecol Reprod Biol. aout 2014;179:36-41.

\section{FIGURE LEGEND}

Fig. 1 The severe outcomes and their occurrence rate in pregnant women with pernicious placenta previa and prior cesarean delivery. Seven hundred and forty-seven pregnant women who had subsequent cesarean delivery due to pernicious placenta previa and prior cesarean history, were selected from 13 hospitals in China. The severe consequences occurred in these patients and their occurrence rates were evaluated.

Table 1 Single factor logistic regression analysis on the potential risk of severe bleeding ([?]1000ml)

\begin{tabular}{|c|c|c|c|c|c|}
\hline factors & $\operatorname{PR}(\%)$ & $\Omega a \lambda \delta \chi^{2}$ & $P$ & OR & $95 \% \mathrm{CI}$ \\
\hline & & & & & lower range \\
\hline delivery times & & 17.104 & $<0.001$ & 2.046 & 1.457 \\
\hline cesarean section times & & 30.252 & $<0.001$ & & \\
\hline $0-1$ & 87.3 & & & 1.000 & \\
\hline 2 & 12.7 & & & 4.985 & 2.812 \\
\hline Interval between previous cesarean section and current deliver (years) & & 5.351 & 0.021 & 0.951 & 0.911 \\
\hline placental implantation under ulreasound & & 55.655 & $<0.001$ & & \\
\hline no & 63.7 & & & 1.000 & \\
\hline yes & 18.9 & & & 3.788 & 2.474 \\
\hline suspecious & 17.4 & & & 3.143 & 2.056 \\
\hline placental implantation under MRI & & 57.059 & $<0.001$ & & \\
\hline no & 16.7 & & & 1.000 & \\
\hline yes & 5.6 & & & 11.892 & 4.846 \\
\hline suspecious & 13.5 & & & 7.230 & 3.996 \\
\hline do not perform MRI & 64.1 & & & 3.047 & 1.993 \\
\hline placental implantation during operation & & 142.683 & $<0.001$ & & \\
\hline no & & & & 1.000 & \\
\hline yes & & & & 9.858 & 6.713 \\
\hline suspecious & & & & 2.241 & 1.466 \\
\hline Type of placenta previa & & 26.573 & $<0.001$ & & \\
\hline marginal & 25.7 & & & 1.000 & \\
\hline partial & 5.1 & & & 1.044 & .516 \\
\hline
\end{tabular}




\begin{tabular}{|c|c|c|c|c|c|}
\hline factors & $\operatorname{PR}(\%)$ & $\Omega a \lambda \delta \chi^{2}$ & $P$ & $O R$ & $95 \%$ CI \\
\hline complete & 69.1 & & & 2.327 & 1.657 \\
\hline
\end{tabular}

Table 2. Consensus analysis of placental abnormality predicted by ultrasound and/or MRI, and intraoperative implantation

\begin{tabular}{|c|c|c|c|}
\hline examination & Abnormal placenta prediction & Placenta implantation by surgery & Placenta implantation by surg \\
\hline \multirow{3}{*}{ Only untrasound } & & Yes (514) & No $(233)$ \\
\hline & Yes $(271)$ & 244 & 27 \\
\hline & No $(476)$ & 270 & 206 \\
\hline \multirow[t]{3}{*}{ Only MRI } & & Yes (178) & No $(90)$ \\
\hline & Yes (143) & 141 & 2 \\
\hline & No $(37)$ & 37 & 88 \\
\hline \multirow[t]{3}{*}{ Untrasound and MRI } & $\mathrm{U}$ and $\mathrm{M}^{*}$ & Yes (178) & No $(90)$ \\
\hline & Yes $(56)$ & 56 & 0 \\
\hline & No $(212)$ & 122 & 90 \\
\hline \multirow[t]{3}{*}{ Untrasound and MRI } & $\mathrm{U}$ or $\mathrm{M}^{*}$ & Yes $(178)$ & No $(90)$ \\
\hline & Yes $(160)$ & 154 & 6 \\
\hline & No $(108)$ & 24 & 84 \\
\hline
\end{tabular}

*U: ultrasound examination; M: MRI examination

Table 3. Relationship between ultrasound- and/or MRI-predicted placenta implantation and adverse outcome of patients $[\mathrm{M}(\mathrm{Ql}, \mathrm{Qu})]$

\begin{tabular}{llll}
\hline items & prediction by ultrasound and MRI & prediction only by ultrasound & prediction only by MRI \\
\hline Bleeding $(\mathrm{ml})$ & $2000(1200-3275)^{\mathrm{a}}$ & $1500(800-2600)^{\mathrm{b}}$ & $1300(800-2575)^{\mathrm{c}}$ \\
Blood transfusion $(\mathrm{ml})$ & $1850(1200-3350)^{\mathrm{a}}$ & $1150(353-2200)^{\mathrm{c}}$ & $1300(258-2140)^{\mathrm{b}}$ \\
with/without hysterectomy & $17 / 39$ & $30 / 187$ & $4 / 86$ \\
\hline
\end{tabular}

Note: a indicates the maximum rank; $b$ indicates the second rank; $c$ indicates the third rank; $d$ indicates the minimum rank time. 


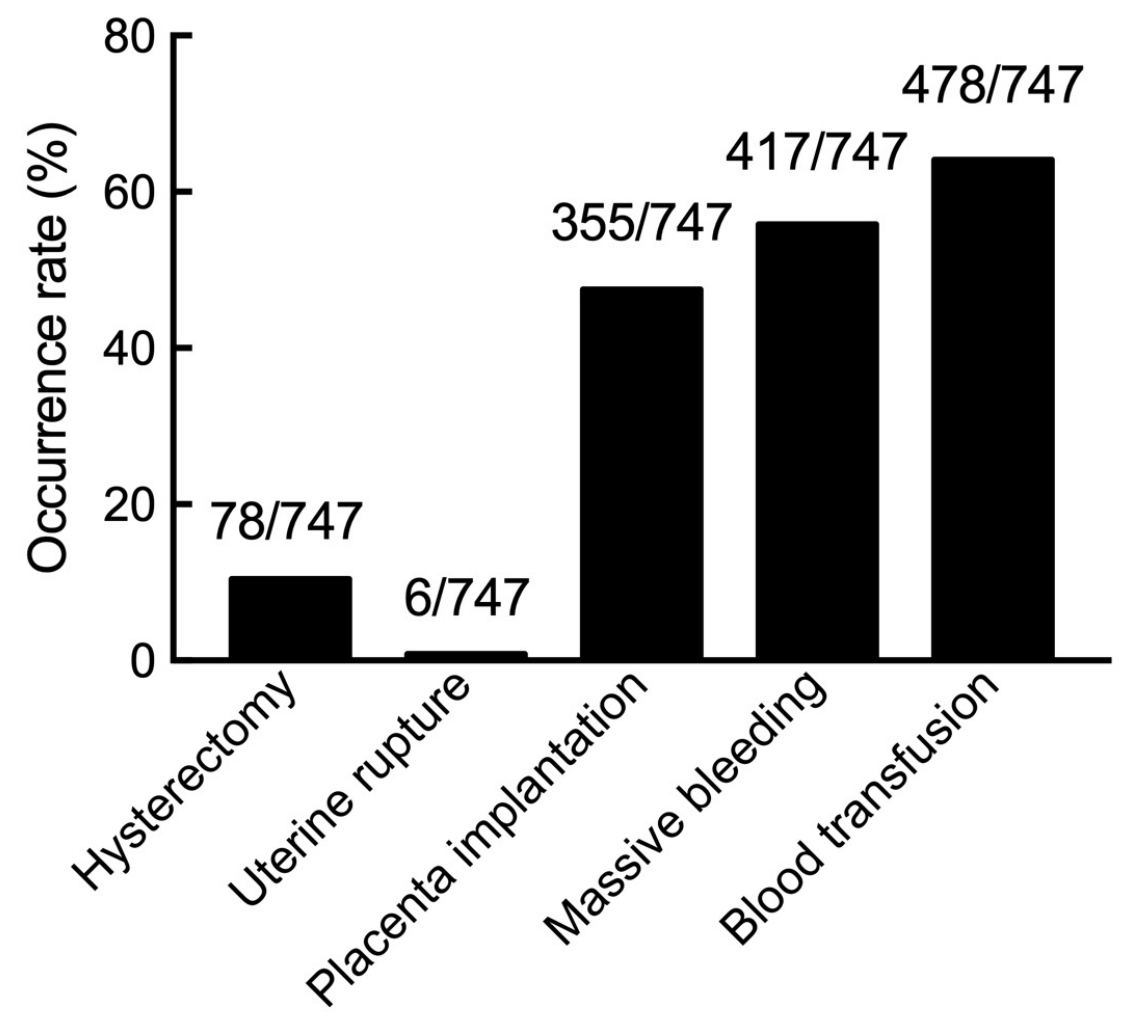

